\title{
Verzeichnis einiger wichtiger Veröffentlichungen auf dem Gebiet der Medizin- und Naturwissenschaftsgeschichte
}

Gustav Scherz, Nicolaus Steno and his Indice. 314 Seiten. Munksgaard, Copenhague 1958.

I. B. Cohen and R. E. Schofield, Isaac Newton's Papers and Letters on Natural Philosophy and Related Documents. 501 Seiten. Harvard University Press, Cambridge (Mass.) 1958.

F. Huber, Daniel Bernoulli (1700-1782) als Physiologe und Statistiker. 104 Seiten. Benno Schwabe, Basel 1958.

Otto Neugebauer, The Exact Sciences in Antiquity. 240 Seiten. Brown University Press, Providence 1957.

Marie Boas, Robert Boyle and Seventeenth-century Chemistry. 240 Seiten. University Press, Cambridge 1958.

F. S. Bodenheimer, The History of Biology. An Introduction. 465 Seiten. Dawson \& Sons, London 1958.

Gh. Singer, A Short History of Anatomy and Physiology from the Greeks to Harvey. 209 Seiten. New-York Dover Publ., 1957.

Circulation. Proceedings of the Harvey Tercentenary Congress held on June 3rdJune 8th 1957 at the Royal College of Surgeons of England, London. Ed. by JoHN McMichael. XXIII+503 Seiten. Blackwell Scientific Publ., Oxford 1958. In the U.S.: Charles C. Thomas, Springfield (Ill.).

Fritz Steckerl, The Fragments of Praxagoras of Cos and his School. 132 Seiten. Brill, Leiden 1958.

A. D. Ritchie, Studies in the History and Methods of the Sciences. University Press, Edinburgh 1958.

Alexander von Humboldt 1769-1859. Gedenkschrift der deutschen Akademie der Wissenschaften zu Berlin. V+471 Seiten. Akademie-Verlag, Berlin 1959.

E. J. Dijksterhuis, Archimedes. 422 Seiten. Ejnar Munksgaard, Copenhague 1956.

H. Schipperges, Honorius und die Naturkunde des 12.Jahrhunderts, Sudhoffs Arch. Gesch. Med. 42 (1958) 71-82.

Avicenne, Le livre de science: II. Physique et mathématiques. Traduit par Mahammad Aschena et H. Massé. Les Belles-Lettres, Paris 1958.

Ibn Sina, Psychologie d'Ibn Sinnā (Avicenne) d'après son œuvre,Aš-Šifa ${ }^{c}$. I : Texte arabe, édité par JAN BAKos, 270 pages; II. Texte arabe édité et traduit en français par JAN BAKOS, 245 pages. Editions de l'Académie tschécoslovaque des Sciences, Prague 1956.

D. P. WALker, Spiritual and Demonic Magic, from Ficino to Campanella. 370 Seiten. Londres 1958. 
J. O. Fleckenstein, G.W. Leibniz. Barock und Universalismus. 200 Seiten. Verlag Ott, Thun/München 1958.

A. Wood, Thomas Young, Natural Philosopher, 1773-1829. University Press, Cambridge 1955.

H. Bеск, Gespräche Alexander von Humboldts. XXXII + 492 Seiten. AkademieVerlag, Berlin 1959.

G. DE Beer, Darwin's Notebooks on Transmutation of Species, Bull. Brit. Museum 2 (1960) $\mathrm{N}^{\circ} 2,25-73,2$ fig.

G. DE BeER, Darwin's Journal, Bull. Brit. Museum 2 (1959) № 1, 1-21.

K.V. Bülow, Charles Darwin als Geologe. Dtsch. Geolog. 8 (1959) 807-817, Porträt.

G. de Beer (Ed.), Some Unpublished Letters of Charles Darwin. Notes. Rec. Roy. Soc. London 14. (1959) $\mathrm{N}^{\circ}$ 1, 12-66, 1 fig., 1 h.t.

A. Lysaght, Some Eighteenth Century Bird Paintings in the Library of Sir Joseph Banks (1743-1820), Bull. Brit. Museum 1 (1959) $\mathrm{N}^{\circ} 6,253-371,3$ h.t., index.

R. J. Pumphrey, The Forgotten Man: Sir John Lubbock. His Contributions to Zoology and his Liberal Record as a Member of Parliament Ought to be Remembered. Science (U.S. A.) 129 (1959) 1087-92, 2 fig.

Irmgard Setzer, Coiters Tabellen der äußeren Körperteile (1564). 35 Seiten. Diss. Würzburg 1958.

J. Brocas, Contribution à l'étude de la vie et de l'œuvre d'André Vésale. 94 pages. R. Foulon, Paris 1958.

William Harvey, traduit par Kenneth J. Franklin, De motu cordis et De circulatione sanguinis. 209 pages et 184 pages. Blackwell Scientific Publ., Oxford 1957 et 1958.

E. Hintzsche, Ein Rollbild altchinesischer Anatomie, Centaurus 6 (1959) $\mathrm{N}^{\circ} 1$, $55-67$.

H. Fischer 